\title{
Intra-nuclear cascade models at low energy?
}

\author{
Y. Yariv ${ }^{1, a}$, Th. Aoust ${ }^{2}$, A. Boudard ${ }^{1}$, J. Cugnon ${ }^{3}$, J.C. David ${ }^{1}$, S. Lemaire ${ }^{1}$, and S. Leray ${ }^{1}$ \\ 1 SPhN, CEA-Saclay, 91191 Gif-sur-Yvette, France \\ 2 SCK-CEN, Boeretang 200, 2400 Mol, Belgium \\ 3 University of Liège, Allée du 6 Août 17, Bât. B5, 4000 Liège 1, Belgium
}

\begin{abstract}
Basic assumptions of the INC models are "revisited" in order to examine their applicability limits at low energies. Details of implementations of INC important at low energies are discussed.
\end{abstract}

\section{Introduction}

The recent activities in production of Rare Isotope Beams and Spallation Sources led to revival of interest in reliable, predictive, simulation of collisions of hadron-nucleus and nucleus-nucleus in the energy range of few tens $\mathrm{MeV}$ to few $\mathrm{GeV}$ per particle to be embedded in transport codes (e.g. MCNPX, GEANT). Owing to the complexity of the quantum-mechanical many-body problems, the processes are often approximately described by Intra-Nuclear Cascade (INC) models followed by de-excitation (sometime twostep) models. INC reproduces successfully wide variety of experimental data of hadron and pion induced reactions, using a small number of adjustable parameters, most with clear physical meaning. INC models have been embedded in the MCNPX transport code, filling the high-energy gap in existing experimental cross-section libraries, which are limited to incident energies of $150 \mathrm{MeV}$ or, for some isotopes, $20 \mathrm{MeV}$. For calculations of residua there is a need to use models already above $20 \mathrm{MeV}$. Understanding of the limitations of INC at low energies is important for evaluation of reliability of transport calculations used in wide variety of applications.

The INC models treat the interaction of incoming projectile with the nucleus as a series of independent collisions using on-mass-shell free particle-nucleon cross sections. The colliding particles are treated as classical point-like objects moving between collisions on well defined trajectories in the target potential well. The collision processes are treated as classical, energy and momentum conserving, scatterings. Collisions violating the Pauli Principle are not allowed - this is the single significant "quantum" property of the model.

\section{Basic assumptions of INC}

In order to determine the applicability of the INC models at low energies we shall "revisit" their basic assumptions [1-3]:

I. The reactions are "deep inelastic" - the energy transferred into internal energy of the target is large in comparison

\footnotetext{
${ }^{a}$ Presenting author, e-mail: yariv@soreq.gov.il

Permanent address: Soreq NRC, Yavne 81800, Israel
}

with the binding energy of nucleons in the target - the many body scattering can be approximately formulated in terms of on-shell single-particle scattering probabilities.

II. The "reduced" de Broglie wavelength, $\bar{\lambda}$, is much smaller than the inter-nucleon distance, $d$. In language of quantum mechanics [4] - the wave-packets representing the particles have good enough definition of position, energy and momentum to be followed on classical trajectories.

III. $\bar{\lambda}$ is much smaller than the mean-free-path between collisions, $\Lambda-$ the scattered wave reaches approximately its asymptotic value before the next scattering, classical treatment of scattering becomes reasonable.

IV. The radius of the target nucleus, $R$, is large with respect to $\Lambda$. There will be many scatterings inside the nucleus and the interference terms between different scattered waves will tend to cancel out.

V. $\Lambda$ is larger than $d$, and the time between interactions, $\Delta \mathrm{t}$, is much longer than the time of an interaction, $T-$ the scattering from different nucleons in the nucleus can be assumed to be approximately independent of each other.

Summarizing, we have:

$$
\begin{gathered}
\bar{\lambda} \ll d<\Lambda<R \\
\Lambda / \beta c>T \approx 10^{-23} \mathrm{sec} \Rightarrow \Lambda / 3 \beta \approx>1 \mathrm{fm} .
\end{gathered}
$$

Figure 1 shows the behaviour of the relevant quantities for proton on ${ }^{208} \mathrm{~Pb}$ reaction as a function of incident proton energy, $E$. Calculating $\bar{\lambda}$ we took into account that proton entering the nucleus gains $\approx 40 \mathrm{MeV}$ kinetic energy.

$$
\bar{\lambda}=\hbar / \sqrt{(E+40)^{2}-m_{p}^{2}},
$$

where $m_{p}$ is the proton mass. The mean free path, $\Lambda$, was calculated using the ISABEL INC code [5] (which includes the Pauli principle) for central collisions:

$$
\Lambda=-2 R / \operatorname{Ln}\left(N_{\text {transp }} / N_{t o t}\right),
$$

where $R=6.63 \mathrm{fm}, N_{\text {transp }}$ is the number of "transparencies" (events when the proton traversed the diameter of the nucleus 


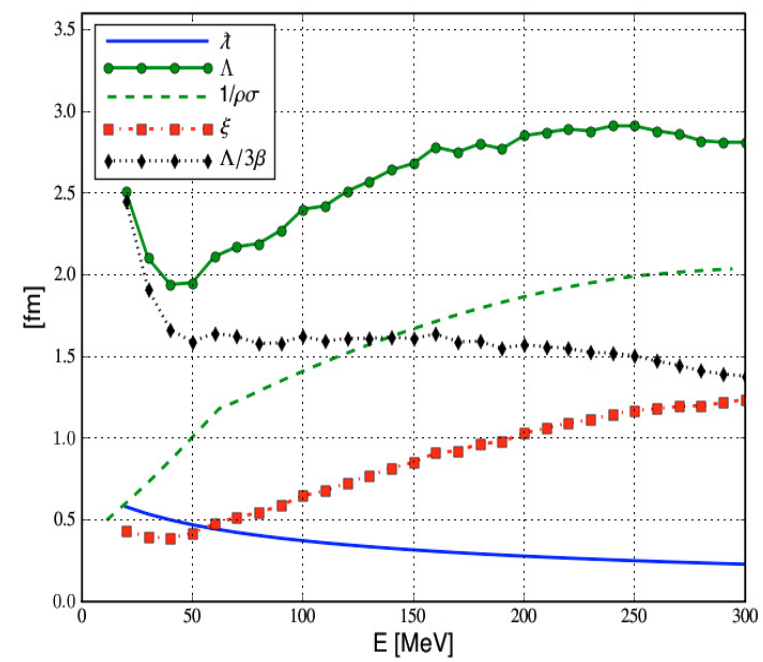

Fig. 1. Central collision proton on ${ }^{208} \mathrm{~Pb}: \bar{\lambda}, \Lambda, \xi=\Lambda / \bar{\lambda} / 10,1 / \rho \sigma$ and $\Lambda / 3 \beta$ as a function of incident proton energy.

without interacting) and $N_{\text {tot }}$ is the total number of events. For comparison also shown is $1 / \rho \sigma$, with $\rho=.16 \mathrm{fm}^{-3}$ being the central nucleon density and $\sigma$ the average proton-nucleon cross section (in $\mathrm{fm}^{2}$ ). This is an estimate of the mean-freepath without the Pauli principle effect.

The effect of Pauli principle is very important. It is especially pronounced at $E_{\text {inc }}<40 \mathrm{MeV}$ causing $\Lambda$ to rise even though the nucleon-nucleon cross section is strongly increasing. In collisions of high energy particle with the Fermi sea, the momentum transfer is small, and Pauli principle limits the interaction to small fraction of the Fermi sea close to its surface, thus increasing the mean free path.

From equation (1a) with $d \approx 2 \mathrm{fm}$, requiring, very conservatively, that $d, \Lambda>5 \bar{\lambda}$, INC is applicable only for $E_{\text {inc }}>$ $60 \mathrm{MeV}$. Requiring $d, \Lambda>10 \bar{\lambda}$, moves the applicability of INC to $E_{\text {inc }}>200 \mathrm{MeV}$. No additional restrictions follow from equation (1b).

Most of the collisions are not central. ISABEL calculations show that in the energies of few tens to few hundreds $\mathrm{MeV}$ about $60 \%$ of the collisions leading to inelastic reactions occur at impact parameters at which the nuclear density is less than a half of the central density. However, in the region of low nuclear density the degenerate Fermi gas potential is low, so the particle gains less kinetic energy entering the nucleus. $50 \mathrm{MeV}$ proton has $\bar{\lambda}=0.6 \mathrm{fm}$ and can not be "localized" on nuclear periphery. On the other hand, proton of $250 \mathrm{MeV}$ has $\bar{\lambda}=0.25 \mathrm{fm}$ and starts to be sensitive to the details of nuclear surface. Still, the lower density of the surface extends the applicability range of INC to lower energies.

It is clear that the applicability range of INC depends strongly on the specific reaction property to be calculated and the desired accuracy.

Thus, considering the total nucleon yields from nucleon induced reactions one may use INC starting from just a few tens of MeV. Using the original Serber's arguments [1], the incident particle will loose in each collision $\approx 10-20 \mathrm{MeV}$. Particle with energies of $\approx<30 \mathrm{MeV}$ will be absorbed in the target nucleus (their probability to interact with the target nucleons is large, and they will "thermalize") unless they are on far periphery of the nucleus. The "thermalized" by INC nucleus has the right excitation energy and momentum (since INC conserves energy and momentum) and may be properly treated by the de-excitation models. In the peripheral collisions the number of emitted particles is expected to be correct in the low energy regime, being determined by energy conservation and the ability of particles to escape the nucleus.

INC may be justified for low energies $\left(E_{\text {inc }} \approx>50 \mathrm{MeV}\right)$ considering reactions which take place primarily on nuclear periphery (e.g., "quasi-elastic", "low multiplicity"). Here, however, the results may strongly depend on the target periphery modelling. We may expect discrepancies, especially when looking in forward direction, due to violation of assumption IV. For "quasi elastic" reactions we may expect distortion of forward angle cross sections due to interference with the elastic channel, which is not accounted for in INC.

Considering "violent" (high multiplicity, high excitation energy) events, which involve the inner parts of the target nucleus the reliability of INC is expected to degrade for energies below $100-200 \mathrm{MeV}$, though it may be used, with caution, according to the original Serber's argument [1].

Until now we have considered the energy limitations on the incident particle. However, an energetic projectile will give little momentum transfer to its Fermi sea collision partners, and create low energy "participants". Inside the nucleus those should be "absorbed" contributing their energy to the excitation of the "remnant" target; on nuclear periphery they may have some chance to escape. The target periphery is modelled in all the INC implementations, but each has a different way to deal with the low energy "participants" chosen considering agreement with the experimental data rather than from basic physical considerations.

High energy cluster $\left(\alpha, \mathrm{d},{ }^{3} \mathrm{He} ..\right)$ production is out of the scope of INC models. In order to calculate those "extra prescriptions" are used. In the "coalescence" model [12,13] the vicinity (configuration, momentum or phase space) of escaping particle is searched for potential particles to share its energy and form a cluster. An alternative "kick-out" process [2] assumes existence of "virtual" clusters in the nucleus which elastically scatter with the cascading particles and then, taking into account their survival probability, escape the nucleus.

\section{Implementations of INC}

The large variety of INC implementations may be divided into two classes: "Time-Like Basis Cascading" (e.g., refs. [2,3, 5-9]) and "Nucleon Dynamics" (e.g., refs. [10,11]). A brief description of the two approaches is given emphasizing the treatment of the low-energy "participants".

\subsection{Time-like basis cascading}

The target nucleus density distribution is represented by a continuous Fermi distribution and the nucleon distribution in the target is assumed to be that of a degenerate Fermi gas in a potential well. It can be shown [9] that under this conditions 
the probability of collision of the incident particle in the interval $\tau$ may be approximated by dividing $\tau$ into $n$ parts and calculating for each $\delta \tau=\tau / n$ the probability of collision with a hypothetical nucleon having momentum $p_{i}$ from degenerate Fermi gas distribution (which depends on the local density):

$$
P_{\text {int }}(\delta \tau) \approx \rho \sigma_{1, i} v_{1, i} \delta \tau
$$

$v_{1, i}$ and $\sigma_{1, i}$ being the relative velocity and scattering cross section of the two particles.

The initial $\delta \tau$ is chosen to be $\Lambda / v n, v$ being the incident particle laboratory velocity, $\Lambda$ is calculated for nucleon density in the centre of the nucleus using the total cross sections of the incoming particle with a stationary protons and neutrons and $n=20-30$.

If the interaction between the incoming particle and a partner from the Fermi sea occurs the calculation of reaction kinematics is performed. Outgoing nucleons ("participants") are checked for Pauli principle violation and only allowed reactions take place. The time interval is updated and the cascading for each of the "participants" continues.

The cascade process stops when all the "participants" leave the target volume or fall below a certain "energy cut-off", close to $E_{F}+E_{B}$ for neutrons and $E_{F}+E_{B}+E_{C}$ for protons, $E_{F}, E_{B}, E_{C}$ being respectively Fermi, binding and Coulomb barrier energies.

The reason for the "energy cut-off" is that the low energy particles are not fulfilling equation (1a) and should be taken care of by the de-excitation models.

\subsection{Nucleon dynamics}

The target nucleus consists of discrete nucleons distributed following Fermi density distribution and degenerate Fermi gas momentum distributions in a potential well. The incident particle and target constituents are moving on classical trajectories in the potential well and scatter whenever their relative distance is less than $\sqrt{\sigma\left(E^{c m}\right) / \pi}, \sigma\left(E^{c m}\right)$ being the free space cross section and $E^{c m}$ their centre of mass energy. Pauli principle violation is not permitted. Particles may reflect on the potential well walls or, reaching the surface with high enough energy, escape the target.

In this approach there is a need to set a restriction on the interaction range or, equivalently, $E_{\min }^{c m}$ - limiting value of $E^{c m}$, below which particles do not scatter. This ensures that low energy particles reaching (or created at) target periphery will be able to escape rather than interact with nuclear interior. $E_{\min }^{c m}$ of $\approx 1925 \mathrm{MeV}$ corresponding to relative lab energy of $\approx 100 \mathrm{MeV}$ (range restriction of $\approx 1.3 \mathrm{fm}$ ) gives generally good results. It is in line with requirements III, V of section 2 . The physical argument behind it is that the long range (or small momentum transfer) interactions are accounted for by the average nuclear potential.

The process stops at a time when properties of the reaction "stabilize" [10] or, alternatively, when all the "participants" left in the target are below certain "energy cut-off" (similar to that used in the "Time-Like Basis" approach).

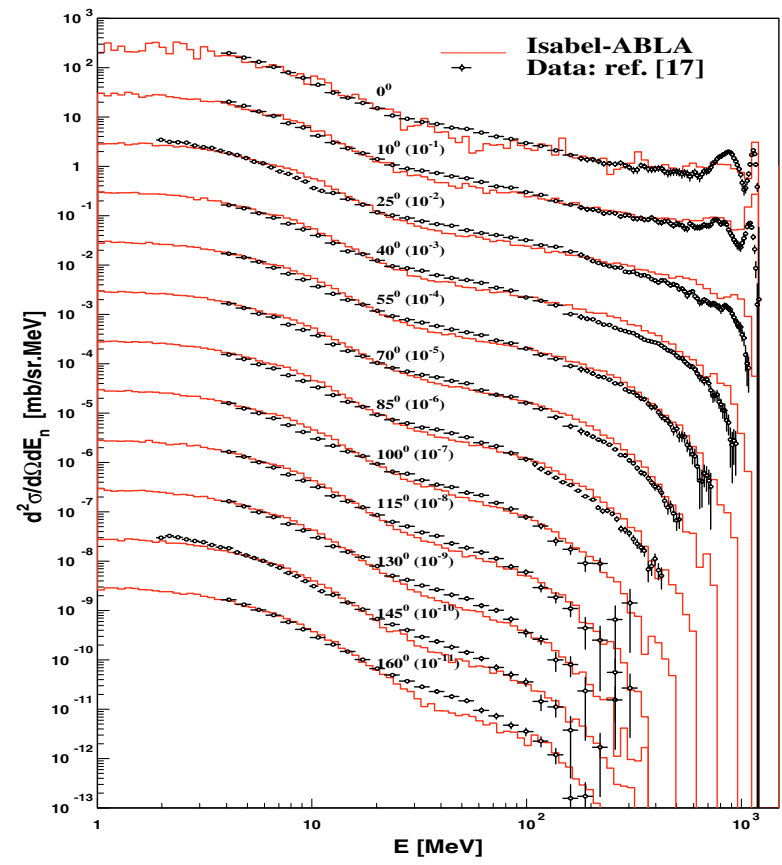

Fig. 2. Neutron double differential cross section for $\mathrm{p}\left({ }^{208} \mathrm{~Pb}, \mathrm{nX}\right)$ reaction at $1.2 \mathrm{GeV}$. INCL4.4 gives very similar results.
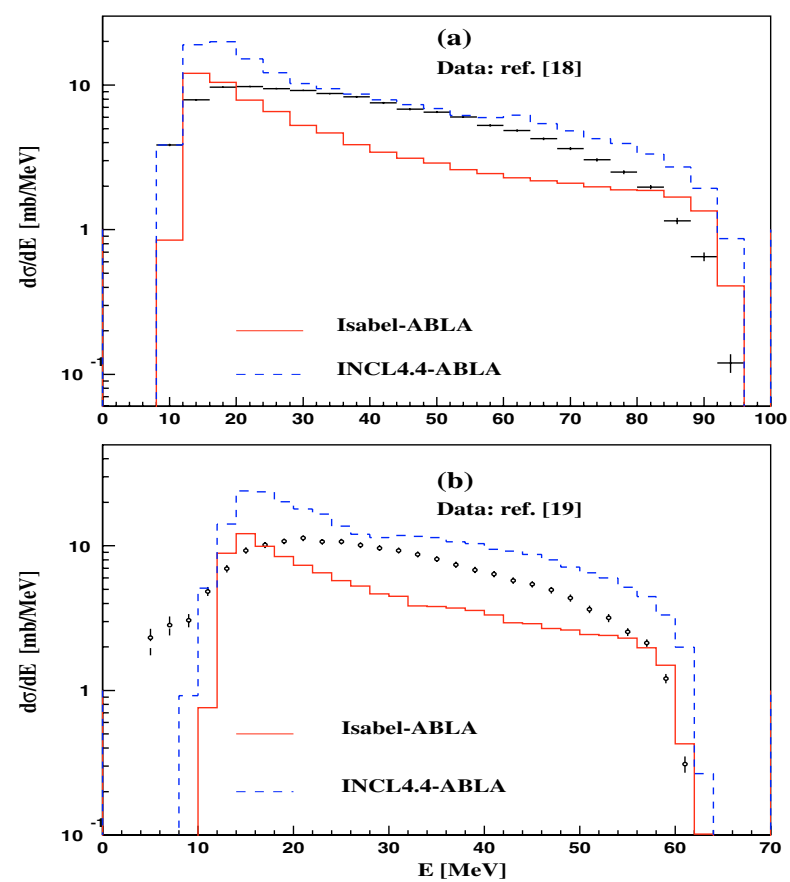

Fig. 3. Proton $d \sigma / d E$ for (a) $\mathrm{n}\left({ }^{208} \mathrm{~Pb}, \mathrm{pX}\right)$ at $96 \mathrm{MeV}$, (b) $\mathrm{n}\left({ }^{209} \mathrm{Bi}, \mathrm{pX}\right)$ at $63 \mathrm{MeV}$.

\section{Examples}

In the following examples the "Time-Like Basis Cascading" is represented by the code ISABEL [5] and the "Nucleon Dynamics" by INCL4.4 [14]. Both are appended with the ABLA de-excitation code $[15,16]$.

Figure 2 shows an example of results INC approach at high energies. Here INCL4 and ISABEL give generally very 


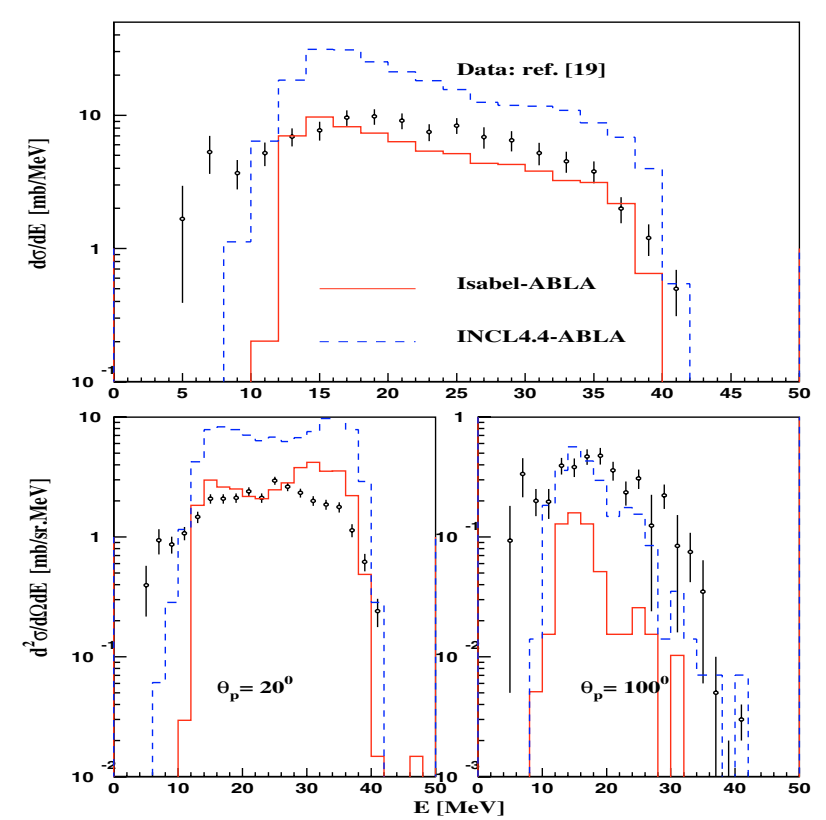

Fig. 4. Proton $d \sigma / d E$ and $20^{\circ}, 100^{0} d \sigma / d E \Omega$ for $\mathrm{n}\left({ }^{209} \mathrm{Bi}, \mathrm{pX}\right)$ at $41 \mathrm{MeV}$.

similar results, and the double differential cross sections are well reproduced. As expected, the biggest discrepancy appears at energies of few tens MeV. This discrepancy grows toward backward angles, where the contribution from scattering of a particle of a massive "collective object" is contributing.

Figures 3 and 4 show that for projectiles of incident energy in the range of $\approx 50-100 \mathrm{MeV}$ the deviation between the models and experimental cross sections may reach factors of 2-5.

At low energies, the calculations are very sensitive to details, such as the momentum distribution of target nucleons on the border of the target, implementation of the Pauli principle and energy cut-offs.

The difference between the models reflects the different method to treat the low energy interactions. At low energies INCL4 gives generally higher $\mathrm{d} \sigma / \mathrm{dE}$ than ISABEL. This is probably due to the $E_{\mathrm{min}}^{c m}$ cut in INCL4 allowing the low energy particles to escape. These particles are captured in ISABEL. Considering $d \sigma / d \Omega d E$ in figure 4: at forward angles, sensitive to the peripheral scattering, ISABEL is closer to experiment, INCL4 being too high probably due to the $E_{\min }^{c m}$ cut; at backward direction ISABEL is underestimating the flux probably due to its extra absorption or lack of contribution from scattering of massive "collective object".
It should be mentioned that neither INCL4 nor ISABEL include "refraction", which was "discredited" for high energy calculations [2,9], but may improve the results for low energies (or even at high energies when using an energy dependent potential [20]).

\section{Conclusions}

INC models coupled to an evaporation-fission model reproduce well experimental data down to $\approx<100 \mathrm{MeV}$. One may expect deviations from experimental data for outgoing particles in the region of few tens $\mathrm{MeV}$.

Allowing for discrepancies, presently of factor $\approx 2-5$, they may be used down to few tens $\mathrm{MeV}$. The actual accuracy depends strongly on specific application, being best for peripheral collisions. At low energies the INC calculations are very sensitive to modelling details leaving space for additional model improvements (e.g., refraction, better treatment of Coulomb force) and possibly phenomenological adjustments.

When using INC for specific applications, one should be aware of its limitations and the resulting uncertainties of the calculations. In parallel there is a place for new, predictive models for the problematic regions.

\section{References}

1. R. Serber, Phys. Rev. 72, 1114 (1947).

2. V.S. Barashenkov, V.D. Toneev, Interaction of High Energy Particles and Atomic Nuclei (Atomizdat, Moscow, 1972), pp. 268-325 (in Russian).

3. J.N. Ginocchio, Phys. Rev. C 17, 195 (1978).

4. A. Messiah, Quantum Mechanics, Vol. I (North-Holland, Amsterdam, 1964), pp. 372-376.

5. Y. Yariv, Z. Fraenkel, Phys. Rev. C 20, 2227 (1979).

6. M.L. Goldberger, Phys. Rev. 74, 1269 (1948).

7. N. Metropolis et al., Phys. Rev. 110, 185 (1958).

8. H.W. Bertini, Phys. Rev. 131, 1801 (1963).

9. K. Chen et al., Phys. Rev. 168, 949 (1968).

10. J. Cugnon, Nucl. Phys. A 462, 751 (1987).

11. A. Boudard et al., Phys. Rev. C 66, 44615 (2002).

12. J. Gosset et al., Phys. Rev. C 16, 629 (1977).

13. A. Boudard et al., Nucl. Phys. A 740, 195 (2004).

14. A. Boudard (these proceedings).

15. J.-J. Gaimard, K.H. Schmidt, Nucl. Phys. A 531, 709 (1991).

16. A.R. Junghans et al., Nucl. Phys. A 629, 635 (1998).

17. S.Leray et al., Phys. Rev. C 65, 44621 (2002).

18. V. Blideanu et al., Phys. Rev. C 70, 14607 (2004).

19. E. Raeymackers et al., Nucl. Phys. A 726, 210 (2003).

20. Th. Aoust et al., Nucl. Instrum. Meth. A 562, 810 (2006). 